\title{
Correction: Polyploidization increases meiotic recombination frequency in Arabidopsis
}

\author{
Ales Pecinka ${ }^{1,2 \dagger}$, Wei Fang ${ }^{3,1+}$, Marc Rehmsmeier ${ }^{4,1}$, Avraham A Levy $^{5}$ and Ortrun Mittelsten Scheid ${ }^{1 *}$
}

\section{Correction}

The authors noted that three values in Table 1 need corrections [1]. The MRF for self-pollinated diploids should be corrected from $15.4 \%$ to $16.8 \%$, for self-pollinated autotetraploids from $20.5 \%$ to $23.2 \%$, and for self-pollinated allotetraploids from $24.1 \%$ to $28.0 \%$, applying the correct formula for genotype combinations upon self-pollination given in reference nine. Similar corrections need to be done to Additional Files 1, 2 and 3. See also Rehmsmeier 2012 [2]. The changes do not affect any of the conclusions presented in the original manuscript. We apologize for any inconvenience caused by this error.

\section{Additional material}

Additional file 1: Additional Table 1

Additional file 2: Additional Table 2.
Additional file 3: Additional Table 3

\section{Author details}

Gregor Mendel Institute of Molecular Plant Biology, 1030 Vienna, Austria. ${ }^{2}$ Max Planck Institute for Plant Breeding Research, Cologne, Germany. ${ }^{3}$ Northwest A \& F University, Shaanxi, P.R. China. ${ }^{4}$ Uni Computing, Bergen, Norway. ${ }^{5}$ Department of Plant Sciences, Weizmann Institute of Science, 76100 Rehovot, Israel.

Received: 3 April 2012 Accepted: 18 April 2012 Published: 18 April 2012

\section{References}

1. Pecinka A, Fang W, Rehmsmeier M, Levy AA, Mittelsten Scheid O:

Polyploidization increases meiotic recombination frequency in Arabidopsis.

BMC Biology 2011, 9:24[http://www.biomedcentral.com/1741-7007/9/24].

2. Rehmsmeier M: Response to Wang and Luo. BMC Biology 2012, 10:32.

doi:10.1186/1741-7007-10-33

Cite this article as: Pecinka et al: Correction: Polyploidization increases meiotic recombination frequency in Arabidopsis. BMC Biology 2012 10:33.

Table 1 Meiotic recombination frequencies (MRF) in diploid, autotetraploid and allotetraploid Arabidopsis ${ }^{1}$

\begin{tabular}{|c|c|c|c|c|c|c|c|c|c|}
\hline \multirow{2}{*}{$\begin{array}{c}\text { Ploidy } \\
\text { (species) }\end{array}$} & \multirow[t]{2}{*}{ Meiosis $^{2}$} & \multirow[t]{2}{*}{ Plants } & \multicolumn{4}{|c|}{ Seed fluorescence } & \multirow[t]{2}{*}{ Seeds total } & \multirow[t]{2}{*}{ MRF (\%) } & \multirow[t]{2}{*}{ S.D. ${ }^{4}(\%)$} \\
\hline & & & Green-only & Red-only & Yellow $^{3}$ & None & & & \\
\hline Diploid & Female & 6 & 66 & 71 & 830 & 894 & 1861 & 7.4 & 1.9 \\
\hline \multirow[t]{2}{*}{ A. thaliana } & Selfed & 3 & 322 & 333 & 2805 & 791 & 4251 & 16.8 & 1.1 \\
\hline & Male & 3 & 147 & 143 & 561 & 582 & 1433 & 20.2 & 0.3 \\
\hline Autotetraploid & Female & 10 & 264 & 317 & 1587 & 1703 & 3871 & 15.0 & 3.2 \\
\hline \multirow[t]{2}{*}{ A. thaliana } & Selfed & 10 & 1868 & 2216 & 12707 & 3098 & 19889 & 23.2 & 1.4 \\
\hline & Male & 9 & 506 & 492 & 1227 & 1345 & 3570 & 28.0 & 3.0 \\
\hline Allotetraploid & Female & 5 & 181 & 214 & 1348 & 1305 & 3048 & 13.0 & 2.5 \\
\hline \multirow[t]{2}{*}{ A. suecica } & Selfed & 5 & 275 & 298 & 1484 & 320 & 2377 & 28.0 & 2.6 \\
\hline & Male & 5 & 598 & 599 & 1412 & 1410 & 4019 & 29.8 & 3.1 \\
\hline
\end{tabular}

\footnotetext{
${ }^{1}$ Detailed values for individual plants are given in Additional Files 1,2 and 3
}

${ }^{2}$ Transmission of the meiotic recombination tester through maternal (female), paternal (male) or both gametes (selfed) determined by reciprocal crosses (female, male) or self-pollination.

${ }^{3}$ Seeds showing both red and green fluorescence.

${ }^{4}$ S.D. - standard deviation, calculated from the individual crosses/self-pollinations in Additional Files 1, 2 and 3

\footnotetext{
* Correspondence: ortrun.mittelsten_scheid@gmi.oeaw.ac.at

† Contributed equally

${ }^{1}$ Gregor Mendel Institute of Molecular Plant Biology, 1030 Vienna, Austria

Full list of author information is available at the end of the article
}

\section{() Biomed Central}

(c) 2012 Pecinka et al; licensee BioMed Central Ltd. This is an Open Access article distributed under the terms of the Creative Commons Attribution License (http://creativecommons.org/licenses/by/2.0), which permits unrestricted use, distribution, and reproduction in any medium, provided the original work is properly cited. 